\title{
Baseline Cortical Thickness Reductions in Clinical High Risk for Psychosis: Brain Regions Associated with Conversion to Psychosis Versus Non-Conversion as Assessed at One-Year Follow-Up in the Shanghai-At-Risk-for-Psychosis (SHARP) Study
}

\author{
Elisabetta C. del Re ${ }^{1,2}$, William S. Stone ${ }^{3}$, Sylvain Bouix ${ }^{2}$, Johanna Seitz ${ }^{2}$, Victor Zeng ${ }^{3}$, Anthony Guliano ${ }^{3}$, \\ Nathaniel Somes ${ }^{2}$, TianHong Zhang ${ }^{4}$, Benjamin Reid ${ }^{2}$, Amanda Lyall ${ }^{2,5}$, Monica Lyons ${ }^{2,5}$, Huijun Li $^{6}$, \\ Susan Whitfield-Gabrieli ${ }^{7}$, Matcheri Keshavan ${ }^{3}$, Larry J. Seidman ${ }^{3,5, \dagger}$, Robert W. McCarley ${ }^{1, \dagger}$, JiJun Wang ${ }^{*, 4}$, \\ Yingying Tang ${ }^{4}$, Martha E. Shenton ${ }^{2,5,8,9}$, and Margaret A. Niznikiewicz ${ }^{* 1,3}$
}

${ }^{1}$ Laboratory of Neuroscience, Department of Psychiatry, VA Boston Healthcare System, Brockton Division, and Harvard Medical School, Boston, MA; ${ }^{2}$ Psychiatry Neuroimaging Laboratory, Department of Psychiatry, Brigham and Women's Hospital, and Harvard Medical School, Boston, MA; ${ }^{3}$ Department of Psychiatry, Beth Israel Deaconess Medical Center, Harvard Medical School, Boston, MA; ${ }^{4}$ Shanghai Mental Health Center, Shanghai Jiaotong University School of Medicine, Shanghai Key Laboratory of Psychotic Disorders, SHARP Program, Shanghai China; ${ }^{5}$ Department of Psychiatry, Massachusetts General Hospital and Harvard Medical School, Boston, MA; ${ }^{6}$ Florida A\&M University, Department of Psychology, Tallahassee, FL; ${ }^{7}$ McGovern Institute for Brain Research, Massachusetts Institute of Technology, Cambridge, MA; ${ }^{8}$ Department of Radiology, Brigham and Women's Hospital, and Harvard Medical School, Boston, MA; ${ }^{9}$ Research and Development, VA Boston Healthcare System, Boston, MA

${ }^{\dagger}$ Deceased.

*To whom correspondence should be addressed; tel: 774-826-2632, e-mail: Margaret_niznikiewicz@hms.harvard.edu

Objective: To assess cortical thickness (CT) and surface area (SA) of frontal, temporal, and parietal brain regions in a large clinical high risk for psychosis (CHR) sample, and to identify cortical brain abnormalities in CHR who convert to psychosis and in the whole CHR sample, compared with the healthy controls (HC). Methods: Magnetic resonance imaging, clinical, and cognitive data were acquired at baseline in $92 \mathrm{HC}, 130$ non-converters, and 22 converters (conversion assessed at 1-year follow-up). CT and SA at baseline were calculated for frontal, temporal, and parietal subregions. Correlations between regions showing group differences and clinical scores and age were also obtained. Results: CT but not SA was significantly reduced in CHR compared with HC. Two patterns of findings emerged: (1) In converters, CT was significantly reduced relative to non-converters and controls in the banks of superior temporal sulcus, Heschl's gyrus, and pars triangularis and (2) $\mathrm{CT}$ in the inferior parietal and supramarginal gyrus, and at trend level in the pars opercularis, fusiform, and middle temporal gyri was significantly reduced in all high-risk individuals compared with HC. Additionally, reduced CT correlated significantly with older age in $\mathrm{HC}$ and in non-converters but not in converters. Conclusions: These results show for the first time that fronto-temporoparietal abnormalities characterized all CHR, that is, both converters and non-converters, relative to $\mathrm{HC}$, while
CT abnormalities in converters relative to CHR-NC and $\mathrm{HC}$ were found in core auditory and language processing regions.

Key words: clinical high risk for psychosis/SA/CT/ language network/prediction of conversion

\section{Introduction}

Structural abnormalities in frontal, temporal, and parietal lobes are common in both early and chronic schizophrenia. ${ }^{1-6}$ Cognitive functions sub-served by these cortices, including language and reasoning, are also affected, ${ }^{7,8}$ as is the progression of these structural abnormalities over the course of illness. ${ }^{4,5,-11}$ Across diagnostic categories, including affective and psychotic illnesses, disruption of a common set of brain regions, together forming the fronto-parietal connectome network has recently been described. ${ }^{12,13}$ Individuals at clinical high risk for developing psychosis (CHR) represent a heterogeneous population, whose clinical profiles are shaped by prodromal symptoms and whose rate of conversion to psychosis is typically between $15 \%$ and $30 \%$, depending in part on whether follow-up assessments were obtained 1,2 , or 3 years past baseline $\left(\mathrm{eg},{ }^{14-17}\right.$ ). We hypothesize that similar to populations with established psychiatric 
diagnoses ${ }^{13}$ who show abnormalities in frontal, temporal, and parietal cortices, CHR will demonstrate abnormalities in these brain regions as well, with some abnormalities characterizing all CHR compared with controls, and other abnormalities characterizing subjects who transition to psychosis (converters: CHR-C) compared with both subjects who do not transition to psychosis (nonconverters: CHR-NC) and healthy controls (HC).

Evidence of brain structural abnormalities is well documented in chronic and first episode schizophrenia, while evidence of such abnormalities is less established for individuals at clinical high risk for developing schizophrenia, although the genetic contribution to schizophrenia suggests a neurodevelopmental component ${ }^{18-23}$ to observed brain changes. Several brain structural magnetic resonance imaging (MRI) studies in CHR report volume reductions in converters, compared with non-converters: CHR-NC. ${ }^{9,24,25}$ Additionally, brain surface contractions in prefrontal cortex ${ }^{26}$ and reductions in gray matter volume of temporal, and parietal cortices, and cingulate gyrus $^{27,28}$ have been observed, but significance of results is limited by small sample sizes and methodological approaches. ${ }^{26,28}$ On the other hand, a somewhat larger CHR study ( 7 converters during a 28 months follow-up) in an Asian population did not find gray matter volume reductions, ${ }^{29}$ nor did a large study involving a North American sample at baseline. ${ }^{30}$ Instead, steeper rates of gray matter reductions in converters compared with non-converters and controls were reported. ${ }^{30}$ Chung et al ${ }^{31}$ grouped CHR into CHR stable/remitted (mild symptoms or remission) and CHR decline (severe symptoms or conversion) with each group further subdivided into younger (12-17 years old) and older (18-35 years old) groups. This approach to CHR categorization yielded evidence of cortical deficits in the younger but not in the older CHR group. While approach allows for constructing larger and more balanced subject groupings, it does not inform about brain differences in relation to conversion, an approach adopted in the current study. Furthermore, Cannon ${ }^{30}$ and Chung ${ }^{31}$ articles are based on data acquired at eight different sites/scanners, in contrast to our study based on one scanning site.

Several recent studies investigating brain structural integrity in CHR have adopted a measure of cortical thickness (CT) $)^{30-34}$ (see Bartholomeusz et $\mathrm{al}^{35}$ for review), while surface area (SA) has only been investigated in just a handful of CHR studies. ${ }^{31,34,36,37}$ Since brain volume is the product of CT and SA, investigating both in the same study makes it possible to evaluate their relative contribution to volumetric brain abnormalities in CHR and tease apart different neurodevelopmental contributions to schizophrenia pathology. Indeed, CT and SA are genetically unrelated, ${ }^{38}$ with distinct maturational stages. ${ }^{39,40}$ In the current study, accordingly, we assessed both CT and SA.
By using a priori defined regions of interests (ROIs), several studies ${ }^{33,41,42}$ have identified CT abnormalities in CHR populations, although some of these studies included no converters ${ }^{41}$ or relied on multisite MRI acquisition and/or low resolution scanners ${ }^{33,42} \mathrm{CT}$ reductions in both prefrontal and parietal cortices have also been identified by using whole brain analysis (eg, Jung et al ${ }^{43}$ [six converters included] and Kwak et $\mathrm{al}^{44}$ [eight converters included], but not all studies report abnormalities. ${ }^{29,30}$

The analytic approach in this study falls between a conservative whole brain analysis and an a priori defined ROI approach. Specifically, we focused on the frontal, temporal, and parietal lobes. ${ }^{12}$ Within these three lobes, we examined all lobe subregions. We were interested in testing the hypothesis that there would exist a set of fronto-temporo-parietal brain abnormalities characterizing the whole at-risk group, as reported in older populations with mixed psychiatric diagnoses, while another set of abnormalities would characterize converters relative to both non-converters and $\mathrm{HC}$ at 1-year clinical follow-up assessment; this hypothesis, to the best of our knowledge, has not been assessed before.

Compared with previous studies, we reduced several sources of heterogeneity and variability. First, this large Chinese sample $(N=244)$, similar to most Asian population studies, had low rates of drug abuse and low dosages of antipsychotic medications, major confounds in detecting subtle structural brain abnormalities. ${ }^{6,29,44,45}$ Second, subject recruitment was based on physician referrals only, thereby ensuring a more homogenous patient sample. ${ }^{44}$ Third, all brain scans were acquired on one MR scanner/one site, thereby reducing variability resulting from the use of different scanners $^{46}$ (eg, Cannon study ${ }^{30}$ (see, eg, harmonization papers and discussions by Mirzaalian et $\mathrm{al}^{47}$ and CetinKarayumak et $\mathrm{al}^{48}$ ). The issue of multiple scanners is of crucial importance for the study of subtle neuroimaging abnormalities.

As brain abnormalities in language-related brain regions occur in prodromal stages ${ }^{20,49,50}$ and language abnormalities are core features of schizophrenia, ${ }^{20,51-53}$ we further hypothesized that primary language regions, as defined by current models of language and current research $^{54-58}$ (see Supplementary figure S1) would be affected in converters. In support of this hypothesis, several recent studies of populations at CHR indicate that features of abnormal language distinguish converters from non-converters ${ }^{50,59,60}$ and schizophrenia from other disorders. ${ }^{61}$

Additionally, we hypothesized that a negative association between age and $\mathrm{CT}$ observed in $\mathrm{HC}$, and indicative of developmentally driven maturation, ${ }^{40}$ would be absent in CHR, suggesting a possible neurodevelopmental component to predicted $\mathrm{CT}$ and SA abnormalities. 


\section{Method}

\section{Participants and Clinical Procedures}

Data for this study were acquired at the Shanghai Mental Health Center (SMHC), Shanghai, China, as part of the ShangHai-At-Risk-for-Psychosis (SHARP) program. Participants included CHR individuals $(n=152)$, of whom 22 were CHR-C: 21 were diagnosed with schizophrenia and 1 with bipolar disorder with psychotic features at a 1 -year clinical follow-up, and 130 were CHR-NC. These subjects were matched to $\mathrm{HC}(n=92)$ for age, gender, and handedness (see table 1). A total of 5 of 22 CHR-C $(22.7 \%)$ and 9/130 CHR-NC (9\%) were medicated with low dose second generation antipsychotics.

Recruitment and clinical assessments, including conversion criteria, are described in detail in $\mathrm{Li}$ et $\mathrm{al}^{17}$ and Zhang et al. ${ }^{62}$ Briefly, the Prodromal Symptoms/Scale of Prodromal Syndromes (SIPS/SOPS), which was validated for use in Chinese samples, ${ }^{63}$ was administered. Subjects who met SIPS diagnostic criteria were classified as CHR. This study was approved by the Human Subjects Review Committees at SMHC and at the Beth Israel Deaconess Medical Center (BIDMC) in Boston. All participants signed an informed consent document prior to study participation.

\section{MRI Acquisition and Parameters}

MR scans were acquired on a Siemens 3T MR B17 (Verio) system, 32-channel head coil, located at SMHC. For the T1-weighted images the following parameters were used: MP-RAGE, repetition time $(\mathrm{TR})=2300 \mathrm{~ms}$, echo time $(\mathrm{TE})=2.96 \mathrm{~ms}$, flip angle $=9$ degree, field of view $(F O V)=256 \mathrm{~mm}$, and voxel size $=1 \mathrm{~mm} \times 1 \mathrm{~mm} \times$ $1 \mathrm{~mm}$ for 192 contiguous sagittal slices.

\section{Image Processing}

Images were visually inspected for possible movement or ghosting artifacts and were then axis realigned and centered. Automatic brain masking was conducted using Multi Atlas Brain Segmentation. ${ }^{64}$ Segmentation of the scans according to the Desikan-Killiany atlas was executed using FreeSurfer $5.3^{65}$ to extract CT and SA. All segmentations were then validated by visual inspection.

\section{Statistical Analyses}

Demographic and clinical variables were analyzed using univariate ANOVAs or $\chi^{2}$ tests (see table 1).

All subregions within each lobe were delineated according to the Desikan-Killiany Atlas. Prior to MRI analyses, data were tested for normality using the ShapiroWilk test. Additionally, CT and SA of each region in the right and left frontal, temporal, and parietal lobes were Z-scored according to appropriate mean and standard deviation of HC. The focus of analyses was on group differences in each of the lobes with secondary focus on hemisphere effects. In the first step, separate analyses were performed for each lobe, that is, for frontal, temporal, and parietal lobe, and separately for CT and for SA. In the analyses, repeated measures MANOVAs were employed with group (HC, CHR-NC, and CHR-C) with gender as between-subject factors, and hemisphere (left and right) and lobe-specific subregions (region) as within-subject factors. Age was used as a covariate in all analyses. The intracranial volume (ICV) was used as covariate in all analyses involving SA variables. The MANOVA for the frontal lobe included 11 sub-regions in each hemisphere: superior (SFG), rostral and caudal middle frontal gyri, the pars opercularis, triangularis, and orbitalis; lateral and medial orbitofrontal cortex, precentral, paracentral gyri, and frontal pole. The MANOVA for the temporal lobe included nine sub-regions, nine in each hemisphere: superior (STG), middle (MTG), and inferior temporal gyri (ITG); banks of the superior temporal sulcus (bSTS); fusiform gyrus (FG); Heschl's gyrus $(\mathrm{HG})$; entorhinal cortex; temporal pole and para-hippocampal cortex. The MANOVA for the parietal lobe included five sub-regions,

Table 1. Group characteristics at baseline

\begin{tabular}{|c|c|c|c|c|c|}
\hline \multirow[b]{2}{*}{ Subjects \# } & \multirow{2}{*}{$\frac{\mathrm{HC}}{92}$} & \multirow{2}{*}{$\frac{\mathrm{CHR}-\mathrm{NC}}{130}$} & \multirow{2}{*}{$\frac{\mathrm{CHR}-\mathrm{C}}{22}$} & \multirow{2}{*}{$\frac{\text { Statistics }}{P\left(F / \chi^{2}\right)}$} & \multirow[t]{2}{*}{ Group Differences } \\
\hline & & & & & \\
\hline Age & $18.8 \pm 4.7$ & $18.7 \pm 4.9$ & $19.5 \pm 5.2$ & .77 & \\
\hline Gender M/F & $46 / 46$ & $61 / 69$ & $15 / 7$ & .183 & \\
\hline Race/ethnicity & Chinese & Chinese & Chinese & & \\
\hline GAF & $80.3 \pm 2.2$ & $54.3 \pm 8.5$ & $52.1 \pm 7.7$ & .001 & $\mathrm{CHR}-\mathrm{C}=\mathrm{CHR}-\mathrm{NC}<\mathrm{HC}$ \\
\hline Medicated/un-medicated & $0 / 92$ & $21 / 109$ & $6 / 16$ & .23 & \\
\hline SOPS total & N/A & $37.2 \pm 10.9$ & $36.6 \pm 11.1$ & .84 & $\mathrm{CHR}-\mathrm{C}=\mathrm{CHR}-\mathrm{NC}$ \\
\hline Positive symptoms & N/A & $10.1 \pm 3.6$ & $10.0 \pm 3.2$ & .86 & $\mathrm{CHR}-\mathrm{C}=\mathrm{CHR}-\mathrm{NC}$ \\
\hline Disorganized symptoms & N/A & $6.53 \pm 3.2$ & $6.5 \pm 2.9$ & .97 & $\mathrm{CHR}-\mathrm{C}=\mathrm{CHR}-\mathrm{NC}$ \\
\hline Negative symptoms & N/A & $11.3 \pm 6.0$ & $11.6 \pm 6.5$ & .84 & $\mathrm{CHR}-\mathrm{C}=\mathrm{CHR}-\mathrm{NC}$ \\
\hline General symptoms & N/A & $9.2 \pm 3.3$ & $8.5 \pm 3.0$ & .41 & $\mathrm{CHR}-\mathrm{C}=\mathrm{CHR}-\mathrm{NC}$ \\
\hline HVLT & $26.2 \pm 3.7$ & $22.8 \pm 5.5$ & $20.9 \pm 4.5$ & $<.001$ & $\mathrm{CHR}-\mathrm{C}=\mathrm{CHR}-\mathrm{NC}<\mathrm{HC}$ \\
\hline BVMT & $30.3 \pm 3.6$ & $27.2 \pm 5.8$ & $24.0 \pm 6.8$ & $<.001$ & $\mathrm{CHR}-\mathrm{C}<\mathrm{CHR}-\mathrm{NC}<\mathrm{HC}$ \\
\hline
\end{tabular}


CT Abnormalities in Clinical High Risk

Table 2. Frontal Lobe: Follow-up MANOVAs Results for Each Region Separately

\begin{tabular}{|c|c|c|c|c|c|}
\hline Effect & $F$ & Hypothesis $d f$ & Error $d f$ & Sig. & Partial $\eta^{2}$ \\
\hline \multicolumn{6}{|l|}{ Superior frontal gyrus } \\
\hline Group & 0.83 & 2.000 & 237.000 & .44 & .007 \\
\hline Hemisphere & 7.3 & 1.000 & 237.000 & .007 & .03 \\
\hline Hemisphere $\times$ Group & 1.1 & 2.000 & 237 & 0.35 & .009 \\
\hline \multicolumn{6}{|c|}{ Rostral middle frontal gyrus } \\
\hline Group & 2.1 & 2.000 & 237.000 & .13 & .01 \\
\hline Hemisphere & 4.3 & 1.000 & 237.000 & .04 & .018 \\
\hline Hemisphere $\times$ Group & 1.8 & 2.000 & 237.000 & .165 & .015 \\
\hline \multicolumn{6}{|l|}{ Caudal middle frontal } \\
\hline Group & .71 & 2.000 & 237.000 & .49 & .006 \\
\hline Hemisphere & 1.7 & 1.000 & 237.000 & .192 & .007 \\
\hline Hemisphere $\times$ Group & 179 & 2.000 & 237.000 & .836 & .002 \\
\hline \multicolumn{6}{|l|}{ Pars opercularis } \\
\hline Group & 3.8 & 2.000 & 237.000 & .024 & .036 \\
\hline Hemisphere & .238 & 1.000 & 237.000 & .626 & .001 \\
\hline Hemisphere $\times$ Group & 2.179 & 2.000 & 237.000 & .115 & .018 \\
\hline \multicolumn{6}{|l|}{ Pars orbitalis } \\
\hline Group & 1.1 & 2.000 & 237.000 & .33 & .004 \\
\hline Hemisphere & 4.99 & 1.000 & 237.000 & .026 & .021 \\
\hline Hemisphere $\times$ Group & .347 & 2.000 & 237.000 & .707 & .003 \\
\hline \multicolumn{6}{|l|}{ Pars triangularis } \\
\hline Group & 5.0 & 2.000 & 237.000 & .007 & .035 \\
\hline Hemisphere & 5.782 & 1.000 & 237.000 & .017 & .024 \\
\hline Hemisphere $\times$ Group & .467 & 2.000 & 237.000 & .628 & .004 \\
\hline \multicolumn{6}{|l|}{ Latero-orbital frontal } \\
\hline Group & 1.1 & 2.000 & 237.000 & .33 & .005 \\
\hline Hemisphere & .110 & 1.000 & 237.000 & .741 & .000463 \\
\hline Hemisphere $\times$ Group & .625 & 2.000 & 237.000 & .536 & .005 \\
\hline \multicolumn{6}{|l|}{ Medial-orbital Frontal } \\
\hline Group & 1.24 & 2.000 & 237.000 & .29 & .012 \\
\hline Hemisphere & 18.83 & 1.000 & 237.000 & .00002 & .074 \\
\hline Hemisphere $\times$ Group & 1.892 & 2.000 & 237.000 & .153 & .016 \\
\hline \multicolumn{6}{|l|}{ Precentral } \\
\hline Group & .26 & 2.000 & 237.000 & .77 & .004 \\
\hline Hemisphere & 2.112 & 1.000 & 237.000 & .147 & .009 \\
\hline Hemisphere $\times$ Group & 3.397 & 2.000 & 237.000 & .035 & .028 \\
\hline \multicolumn{6}{|l|}{ Paracentral } \\
\hline Group & 0.485 & 2.000 & 237.000 & .62 & .007 \\
\hline Hemisphere & .59 & 1.000 & 237.000 & .443 & .002 \\
\hline Hemisphere $\times$ Group & .634 & 2.000 & 237.000 & .531 & .005 \\
\hline \multicolumn{6}{|l|}{ Frontal Pole } \\
\hline Group & 1.67 & 2.000 & 237.000 & .19 & .012 \\
\hline Hemisphere & 28.3 & 1.000 & 237.000 & .00001 & .1 \\
\hline Hemisphere $\times$ Group & .374 & 2.000 & 237.000 & .689 & .003 \\
\hline
\end{tabular}

in each hemisphere: superior (SPG), inferior (IPG), supramarginal (sMG), postcentral gyri (pCG), and the precuneus. Bonferroni correction was entered into each MANOVA model.

In the second step of analyses, we followed up on all significant interactions involving group: between group and region, or between group, gender, and region. Accordingly, repeated measures MANOVAs with group or group and gender as independent variables and hemisphere as a within-subjects factor were carried out for each subregion of each lobe separately. Bonferroni correction was entered into each MANOVA model.
Where group differences were identified, pairwise comparisons were used to examine group differences among three groups in a given region. Bonferroni corrections were applied to correct for multiple comparisons.

Correlational Analyses. For clinical-structural MRI correlations, positive, negative, general, and disorganization scores from the Structured Interview for Prodromal Syndromes (SIPS) ${ }^{63,66}$ were used. Age was controlled for, and Spearman's $r$ values are reported.

Correlations between age and CT were also assessed for CHR-C and CHR-NC separately given the different statistical results obtained for these two groups (SA was not included given a lack of significant group differences). 
Table 3. Temporal Lobe: Follow-up MANOVAs Results for Each Region Separately

\begin{tabular}{|c|c|c|c|c|c|}
\hline Effect & $F$ & Hypothesis $d f$ & Error $d f$ & Sig. & Partial $\eta^{2}$ \\
\hline \multicolumn{6}{|l|}{ Superior temporal gyrus } \\
\hline Group & 2.6 & 2.000 & 240.000 & .075 & .121 \\
\hline Hemisphere & 1.186 & 1.000 & 240.000 & .277 & .005 \\
\hline Hemisphere $\times$ Group & .547 & 2.000 & 240.000 & .005 & .019 \\
\hline \multicolumn{6}{|l|}{ Middle temporal gyrus } \\
\hline Group & 5.9 & 2.000 & 240.000 & .003 & .049 \\
\hline Hemisphere & 6.813 & 1.000 & 240.000 & .01 & .028 \\
\hline Hemisphere $\times$ Group & 2.283 & 2.000 & 240.000 & .104 & .019 \\
\hline \multicolumn{6}{|l|}{ Inferior temporal gyrus } \\
\hline Group & 2.8 & 2.000 & 240.000 & .06 & .024 \\
\hline Hemisphere & .294 & 1.000 & 240.000 & .588 & .001 \\
\hline Hemisphere $\times$ Group & 1.477 & 2.000 & 240.000 & .230 & .012 \\
\hline \multicolumn{6}{|c|}{ Banks of the superior temporal sulcus } \\
\hline Group & 4.4 & 2.000 & 240.000 & .013 & .037 \\
\hline Hemisphere & .371 & 1.000 & 240.000 & .543 & .002 \\
\hline Hemisphere $\times$ Group & .309 & 2.000 & 240.000 & .734 & .003 \\
\hline \multicolumn{6}{|l|}{ Fusiform } \\
\hline Group & 4.6 & 2.000 & 240.000 & .01 & .039 \\
\hline Hemisphere & .007 & 1.000 & 240.000 & .934 & .00003 \\
\hline Hemisphere $\times$ Group & .758 & 2.000 & 240.000 & .47 & .006 \\
\hline \multicolumn{6}{|l|}{ Heschl gyrus } \\
\hline Group & 6.1 & 2.000 & 240.000 & .003 & .047 \\
\hline Hemisphere & 1.224 & 1.000 & 240.000 & .27 & .005 \\
\hline Hemisphere $\times$ Group & .234 & 2.000 & 240.000 & .792 & .002 \\
\hline \multicolumn{6}{|l|}{ Entorhinal cortex } \\
\hline Group & .73 & 2.000 & 240.000 & .48 & .006 \\
\hline Hemisphere & 3.079 & 1.000 & 240.000 & .08 & .013 \\
\hline Hemisphere $\times$ Group & .57 & 2.000 & 240.000 & .566 & .005 \\
\hline \multicolumn{6}{|l|}{ Temporal pole } \\
\hline Group & 2.116 & 2.000 & 240.000 & .123 & .017 \\
\hline Hemisphere & .738 & 1.000 & 240.000 & .391 & .003 \\
\hline Hemisphere $\times$ Group & 1.172 & 2.000 & 240.000 & .311 & .01 \\
\hline \multicolumn{6}{|c|}{ Para-hippocampal cortex } \\
\hline Group & .14 & 2.000 & 240.000 & .87 & .001 \\
\hline Hemisphere & 6.207 & 1.000 & 240.000 & .013 & .025 \\
\hline Hemisphere $\times$ Group & 1.243 & 2.000 & 240.000 & .29 & .01 \\
\hline
\end{tabular}

\section{Results}

\section{Participants' Group Characteristics}

The three groups did not differ in age or gender, but CHR-C and CHR-NC Global Assessment of Functioning (GAF) scores were significantly lower than $\mathrm{HC}$, as expected (see table 1; see Supplementary materials for morphometric analyses as a function of GAF scores).

\section{Imaging Results}

Main imaging results are summarized in tables 2-5; and further in Supplementary table S1A-C.

\section{CT Analyses}

Frontal Lobe. The omnibus MANOVA with 11 subregions showed a significant interaction of Group by Region by Gender $\left[F(20,456)=1.7 ; P=.016 ; \eta^{2}=0.07\right]$. The follow-up MANOVAs demonstrated that this significant interaction was driven by significant CT group differences in the pars triangularis and the pars opercularis (see table 2).

Pairwise comparisons showed that CT of pars triangularis in converters was significantly lower than in non-converters $(P=.0073$; corrected $P=.022)$ and in HC $(P=.012$; corrected $P=.036)$. No significant difference was found between non-converters and $\mathrm{HC}$ (see table 5). Pars Opercularis. CT in converters was significantly lower than in HC $(P=.012$, corrected $P=.036)$ but was not different than in non-converters $(P=.18$; corrected $P=.54)$, with a trend level CT reduction in nonconverters relative to $\mathrm{HC}(P=.023$; corrected $P=.076$; table 5).

Frontal lobe CT hemisphere effects identified in follow-up MANOVAs:

Several regions showed larger left than right hemisphere, without significant group interactions (see table 2).

Temporal Lobe. The omnibus MANOVA with nine regions showed that there was a main effect of group 
Table 4. Parietal Lobe: Follow-up MANOVAs Results for Each Region Separately

\begin{tabular}{|c|c|c|c|c|c|}
\hline Effect & $F$ & Hypothesis $d f$ & Error $d f$ & Sig. & Partial $\eta^{2}$ \\
\hline \multicolumn{6}{|l|}{ Superior parietal gyrus } \\
\hline Group & 1.916 & 2.000 & 240.000 & .15 & .015 \\
\hline Hemisphere & 1.940 & 1.000 & 240.000 & .165 & .008 \\
\hline Hemisphere $\times$ Group & .138 & 2.000 & 240.000 & .871 & .001 \\
\hline \multicolumn{6}{|l|}{ Inferior parietal gyrus } \\
\hline Group & 7.64 & 2.000 & 240.000 & .001 & .063 \\
\hline Hemisphere & .553 & 1.000 & 240.000 & .458 & .002 \\
\hline Hemisphere $\times$ Group & .29 & 2.000 & 240.000 & .749 & .002 \\
\hline \multicolumn{6}{|l|}{ Supramarginal } \\
\hline Group & 6.33 & 2.000 & 240.000 & .002 & .053 \\
\hline Hemisphere & 4.485 & 1.000 & 240.000 & .035 & .018 \\
\hline Hemisphere $\times$ Group & .331 & 2.000 & 240.000 & .718 & .003 \\
\hline \multicolumn{6}{|l|}{ Postcentral } \\
\hline Group & 1.96 & 2.000 & 240.000 & .143 & .015 \\
\hline Hemisphere & .907 & 1.000 & 240.000 & .342 & .004 \\
\hline Hemisphere $\times$ Group & 1.526 & 2.000 & 240.000 & .219 & .013 \\
\hline \multicolumn{6}{|l|}{ Precuneus } \\
\hline Group & 2.69 & 2.000 & 240.000 & .02 & .022 \\
\hline Hemisphere & 1.945 & 1.000 & 240.000 & .164 & .008 \\
\hline Hemisphere $\times$ Group & .432 & 2.000 & 240.000 & .650 & .004 \\
\hline
\end{tabular}

Table 5. Post Hoc Comparisons

\begin{tabular}{|c|c|c|c|c|c|c|}
\hline Group Differences ${ }^{\mathrm{a}}$ & $\mathrm{HC}$ vs CHR-NC & Cohen's $d^{b}$ & $\mathrm{HC}$ vs CHR-C & Cohen's $d^{c}$ & $\begin{array}{l}\text { CHR-NC vs } \\
\text { CHR-C }\end{array}$ & Cohen's $d^{\mathrm{d}}$ \\
\hline \multicolumn{7}{|l|}{$\begin{array}{l}\text { Converters-only CT } \\
\text { abnormalities }\end{array}$} \\
\hline Pars Triangularis & $P=.99(0.33)$ & $d=0.03$ & $P=.036(0.012)$ & $d=0.72$ & $P=.022(0.0073)$ & $d=0.72$ \\
\hline Banks of STS & $P=.72(0.24)$ & $d=0.17$ & $P=.024(0.008)$ & $d=0.74$ & $P=.048(0.016)$ & $d=0.6$ \\
\hline Heschl Gyrus & $P=.3(0.1)$ & $d=0.14$ & $P=.006(0.002)$ & $d=0.96$ & $P=.036(0.012)$ & $d=0.6$ \\
\hline \multicolumn{7}{|l|}{$\begin{array}{l}\text { CT abnormalities } \\
\text { common to All CHR }\end{array}$} \\
\hline Pars Opercularis & $P=.075(0.025)$ & $d=0.3$ & $P=.036(0.012)$ & $d=0.6$ & $P=.54(.18)$ & $d=0.26$ \\
\hline Supramarginal & $P=.03(0.01)$ & $d=0.7$ & $P=.003(0.001)$ & $d=0.76$ & $P=.18(0.06)$ & $d=0.38$ \\
\hline Inferior Parietal & $P=.006(0.002)$ & $d=0.4$ & $P=.002(0.0007)$ & $d=0.7$ & $P=.33(0.1)$ & $d=0.3$ \\
\hline MTG & $P=.07(0.023)$ & $d=0.3$ & $P=.003(0.001)$ & $d=0.8$ & $P=.135(0.045)$ & $d=0.3$ \\
\hline Fusiform & $P=.072(0.024)$ & $d=0.3$ & $P=.018(0.006)$ & $d=0.63$ & $P=.42(0.14)$ & $d=0.3$ \\
\hline
\end{tabular}

${ }^{\text {a}}$ For each post hoc comparison, uncorrected $P$ values are listed in parenthesis.

${ }^{\mathrm{b}}$ For all, $\mathrm{HC}>\mathrm{CHR}-\mathrm{NC}$.

${ }^{\mathrm{c}}$ For all, $\mathrm{HC}>\mathrm{CHR}-\mathrm{C}$.

${ }^{\mathrm{d}}$ For all, CHR-NC $>$ CHR-C.

$\left[F(2,237)=3.82 ; P=.023, \eta^{2}=0.031\right]$ with lower temporal lobe $\mathrm{CT}$ in converters compared with $\mathrm{HC}(P=.02$; corrected $P=.06$ ) and $\mathrm{CT}$ of non-converters not different from converters $(P=.11$; corrected $P=.33)$ or from $\mathrm{HC}$ $(P=.65$; corrected $P=1.00)$. The interaction between Region and Group was significant $[F(16,460)=2.1$; $\left.P=.007, \eta^{2}=0.07\right]$.

The follow-up MANOVAs showed that the interaction was driven by four regions, including Heschl gyrus, the banks of the superior temporal sulcus, the middle temporal gyrus, and the fusiform gyrus (see table 3).

Pairwise comparisons showed that CT in converters was lower than in non-converters and $\mathrm{HC}$ for the Heschl gyrus and the banks of the superior temporal sulcus; while CT of non-converters and $\mathrm{HC}$ did not differ (see table 5).

$\mathrm{CT}$ of the middle temporal and the fusiform gyri was equivalent in converters and non-converters, and lower than in $\mathrm{HC}$ (see table 5).

Temporal lobe CT hemisphere effects identified in follow-up MANOVAs:

The middle temporal gyrus and the parahippocampal cortex had greater left than right CT in all groups (see table 3).

Parietal Lobe. The omnibus MANOVA with five regions showed a main effect of group $[F(2,237)=3.8, P$ 
$\left.=.024 ; \eta^{2}=0.031\right]$ with smaller $\mathrm{CT}$ in converters relative to $\mathrm{HC}(P=.013$; corrected $P=.04)$, while $\mathrm{CT}$ of non-converters did not differ from converters $(P=.047$; corrected $P=.14)$ or HC $(P=.21$; corrected $P=.63)$. There was also a significant interaction between Region and Group $\left[F(8,468)=2.16, P=.03, \eta^{2}=0.036\right]$.

Follow-up MANOVAs showed that the interaction was driven by significant $\mathrm{CT}$ differences of the inferior parietal and the supra-marginal gyri (see table 4).

Pairwise comparisons showed that CT of the inferior parietal did not differ between converters and nonconverters but was lower than in $\mathrm{HC}$ (table 5). CT of the supra-marginal gyrus did not differ in converters and non-converters but was lower than in $\mathrm{HC}$ (table 5).

Parietal lobe CT hemisphere effects identified in follow-up MANOVAs.

The CT of the supra-marginal gyrus, inferior parietal gyrus, and precuneus was greater in the left hemisphere in all groups (see table 4 ).

\section{SA Analysis}

Lobes. SA did not differ statistically among the three groups for any of the three lobes (frontal lobe $[F(2,241)=1.0 ; P=.37]$, temporal lobe $[F(2,241)=0.45$; $P=.64]$, or parietal lobe $[F(2,237)=2.3, P=.1])$.

\section{Correlational Analyses}

Relationship Between Age and CT. We explored the relationship between age and CT separately in CHR-C, CHR-NC, and $\mathrm{HC}$ in the eight brain regions that showed statistically significant group differences. CT for all regions, with the exception of $\mathrm{HG}$, was strongly and inversely correlated with age in both $\mathrm{HC}$ and CHR-CN. That is, older age was associated with reduced CT. In contrast, no statistically significant correlations were found in the CHR-C group (see figure 2 and Supplementary table S2).

Clinical Variables. No significant correlations were found between CT and SIPS scores.

\section{Discussion}

This examination of CT and SA in frontal, temporal, and parietal lobes in a large group $(N=152)$ of individuals at $\mathrm{CHR}$ revealed that $\mathrm{CT}$, but not $\mathrm{SA}$, was reduced in $\mathrm{CHR}$. As volume is the product of $\mathrm{CT}$ and SA, these results suggest that $\mathrm{CT}$ is the major contributor to reported volume reductions in CHR. Notably, this is the first report to identify both a set of $\mathrm{CT}$ abnormalities that characterizes converters and non-converters (ie, the whole CHR sample), and another set of abnormalities that characterizes converters relative to non-converters and $\mathrm{HC}$ (see figure 1).

Individuals at CHR represent a diverse population, where only up to a third of individuals will convert to psychosis. ${ }^{17}$ In populations with established, mixed psychiatric diagnoses, functional connectivity studies have identified abnormalities in a set of regions involving the frontal, temporal and parietal cortices, and the frontoparietal network. . $^{12,13,67}$

In this study, the CHR group irrespective of outcome, as assessed at 1-year follow-up, showed CT abnormalities largely overlapping with the fronto-parietal network described in functional connectivity studies ${ }^{68}$ of mixed psychiatric populations. ${ }^{12,13}$ The converters to psychosis, relative to both non-converters and $\mathrm{HC}$, showed $\mathrm{CT}$ abnormalities in the Heschl gyrus, the banks of the superior temporal sulcus and the pars triangularis. These regions are regarded as the core of the language network. ${ }^{54-58}$

Baseline CT reductions found in the current study contrast with two other large CHR studies of European ancestry $^{29,30}$ but agree with the large study of Asian CHR, ${ }^{44}$ although this study lacks comparisons between converters and non-converters. Possible reasons for the lack of CT reductions ${ }^{29,30}$ may include a low number of converters $\left(\mathrm{eg},{ }^{29}\right)$ and the use of multiple scanners ${ }^{30}$ which might make accurate measurements of smaller cortical regions more challenging. As discussed above, Chung et al ${ }^{31}$ study reported CHR-HC differences across a number of measures but did not examine converter-non-converter status relative to $\mathrm{HC}$, which complicates direct comparisons with the current study.

Thus, the different findings may reflect heterogeneity introduced by recruitment methods, ${ }^{16,17,29,44,69}$ smaller subject cohorts and the utilization of multiple scanners (eg, $\left.{ }^{30,36,46,70,71}\right)$. In the current large Asian study, MR data were acquired on one scanner at one acquisition site using a large $\mathrm{CHR}$ cohort with minimal exposure to drug abuse and with limited use of antipsychotic medication. Furthermore, all subjects were accepted into the study based on physician referral, which reduces sampling variability. Thus, most common sources of variability were reduced in this study.

Two important findings emerge from this study. (1) Cortical thinning, at baseline, in all CHR, that is, irrespective of the conversion status, was observed in the frontotemporo-parietal network including in: (a) the frontal lobe, where CT reductions were found in the pars opercularis, (b) the temporal lobe, where CT reductions were found in the middle temporal and the fusiform gyri, and (c) the parietal lobe, with CT reductions in the supramarginal and the inferior parietal gyri (see figure 1). (2) CT reductions in CHR-C relative to both $\mathrm{HC}$ and CHR-NC were observed in the frontal (pars triangularis) and temporal regions (Heschl gyrus and banks of superior temporal sulcus; see figure 1). We interpret these regions as primary auditory and core language regions following the current literature (eg, ${ }^{54,55,72-74}$ also see Supplementary figure S1).

The status of conversion was assessed at 1-year clinical follow-up. Thus, observed CT reductions in CHR-C at baseline may serve as markers of transition to psychosis, while CT abnormalities observed in all CHR 


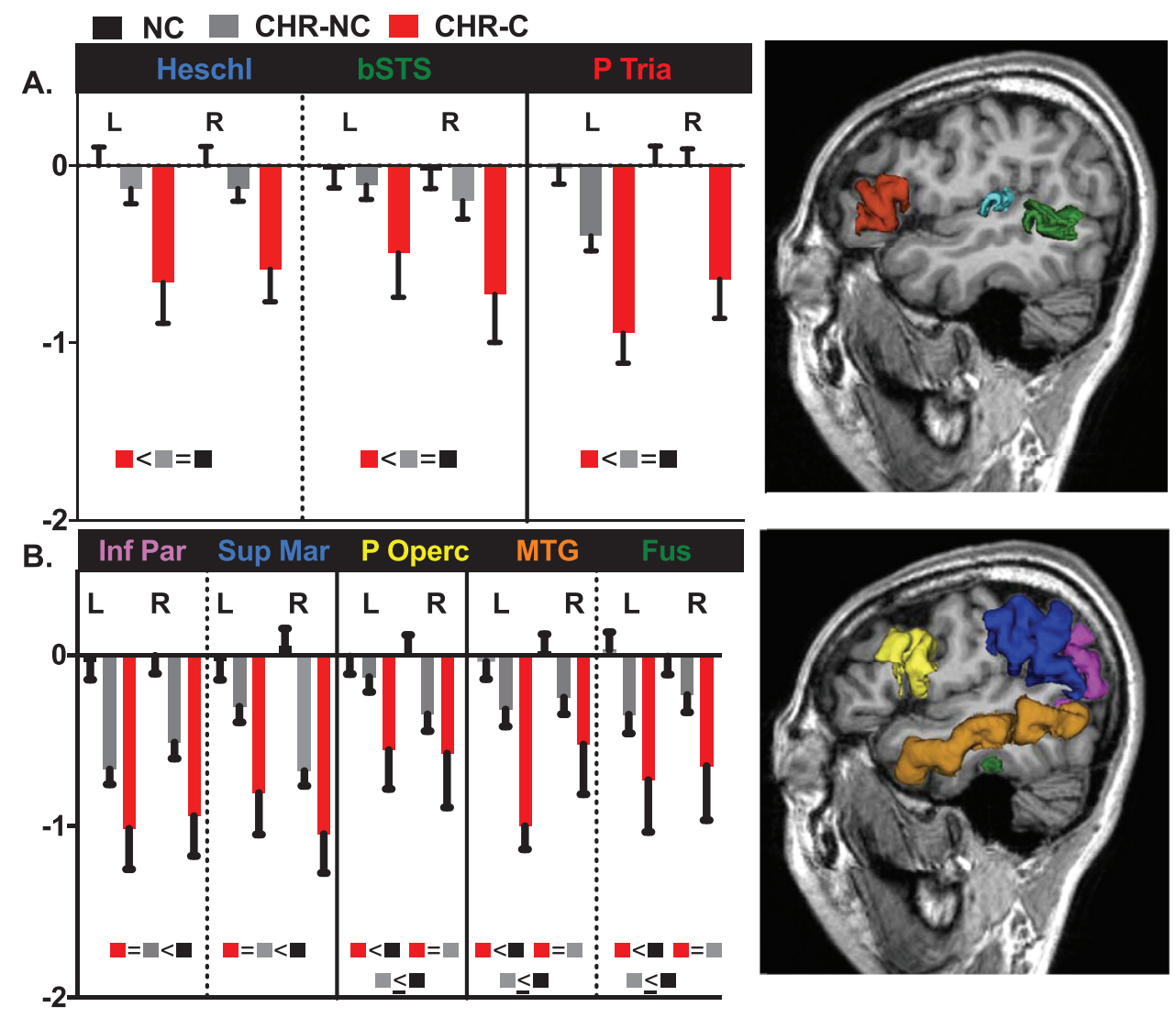

Fig. 1. CT differences between $\mathrm{HC}$ and converters and non-converters for all regions where there was a significant interaction of Diagnosis $\times$ Region. (A) The group differences are depicted for brain regions affected in converters only and (B) brain regions affected in the whole CHR sample. Z-scores of CT in healthy controls (HC; black bar), non-converters (CHR-NC; gray bar), and converters (CHR$\mathrm{C}$; red bar) in left and right hemisphere (R, right hemisphere; L, left hemisphere). Heschl's gyrus (light blue color); banks of the superior temporal sulcus (bSTS, green color); pars triangularis (PTria, red color); Inferior parietal (Inf Par, pink color); supramarginal (Sup Mar, blue color); pars opercularis (P Operc, yellow color); middle temporal gyrus (MTG, orange color); fusiform (Fus, deep green color). See Method section for $P$ values.

(ie, both converters and non-converters) might serve as markers of high-risk status, irrespective of conversion. CT reductions in converters relative to both nonconverters and $\mathrm{HC}$ affected regions including the pars triangularis, the Heschl gyrus and the banks of the superior temporal sulcus, which are involved in auditory processing ${ }^{75}$ speech (including prosody processing) ${ }^{75}$ and semantic and syntactic processing. ${ }^{76,77}$ Thus, conversion to psychosis was related to CT reductions in primary auditory and core portions of language regions. These findings implicating language-related brain regions in CHR are novel but dovetail nicely with recent functional MRI studies in CHR that identified abnormalities $^{78-80}$ in several brain regions where CT reductions were observed in the current study.

The regions affected in the whole CHR group (ie, converters + non-converters) spanned the frontal, the temporal, and the parietal lobe. These fronto-temporoparietal brain subregions are involved in an array of cognitive functions and are activated according to the cognitive task at hand. ${ }^{68,81-83}$ Functions that have been ascribed to these regions include semantic, phonological, and orthographic processes (IPG and FG) ${ }^{84}$ multimodal association functions involved in word reading, comprehension, and semantic analyses (angular gyrus; see Seghier ${ }^{85}$ ), interfacing between phonetic and articulatory representations (sMG; $\mathrm{Gow}^{86}$ ), and between phonetic and semantic representations (MTG). Furthermore, the fronto-temporo-parietal regions contribute to cognitive flexibility: self-agency processing ${ }^{87}$ working memory processes, ${ }^{88}$ bottom-up attention, undirected thinking, episodic memory, and social cognition (see ref. ${ }^{89}$ for a review), delayed reward discounting, ${ }^{90}$ theory of mind ${ }^{91}$ (important for facilitating the development of inferences about complex linguistic messages), and face processing. ${ }^{92,93}$ Notably, all of these functions make full and flexible use of human language possible. ${ }^{94}$

Furthermore, our data cautiously support the hypothesis of abnormal neurodevelopmental CT trajectories in CHR. More specifically, significant negative relationships were observed between $\mathrm{CT}$ and age in $\mathrm{HC}$ and in CHR-NC in several of the regions where $\mathrm{CT}$ reductions 


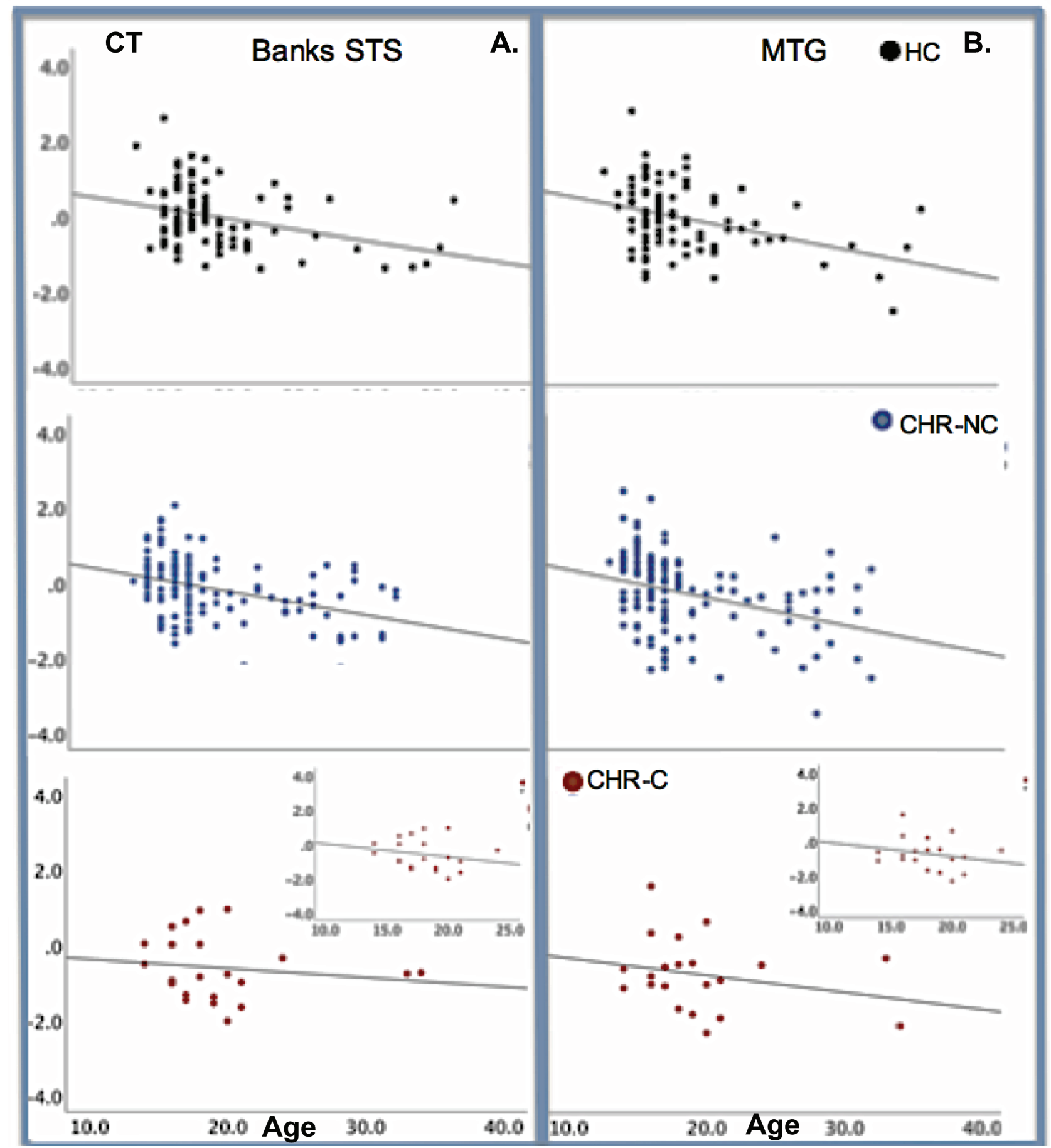

Fig. 2. Correlations between CT and age. (A) Correlations between the bilateral banks of the superior temporal sulcus (bSTS) and age; (B) Correlations between the middle temporal gyrus (MTG) and age. In both healthy controls (HC; top panels, dots) and in nonconverters (CHR-NC; middle panels), there is a significant negative correlation between age and CT, that is older age is associated with smaller CT. This negative correlation is interpreted as an index of maturation processes. In converters (CHR-C; lower panels), the correlation between CT and age is not significant. The insert in the CHR-C panels depict the correlation in CHR-C between 12 and 25 years of age to demonstrate that eliminating the two CHR-C older subjects does not change the result. Least square fit lines are drawn. Pearson's $r$ and probability values are as indicated.

were observed (age range: HC:14-34; CHR-NC: 13-32). Importantly, this relationship (indicative of a neurotypical development) was absent in converters to psychosis (age range 13-36; see figure 2).
Cortical thinning is characteristic of brain maturation as a function of age ${ }^{40,95,96}$ and likely results from multiple neurodevelopmental processes such as pruning, ${ }^{97}$ myelination, and cortical morphology. ${ }^{98}$ Pruning is believed 
to reflect prolonged fine-tuning of neuronal connections that extends beyond adolescence in typically developing individuals, ${ }^{40,96,99,100}$ while myelination indicates growth of myelin sheaths around axons, a process that in traditionally analyzed MRI appears as thinning of the gray matter at the gray/white matter boundary. Increased gyrification/ cortical folding might also explain the apparent perceived thinning of the cortex during development. ${ }^{101}$ The three possibilities are not mutually exclusive, and our data in relation to age might result from any of these processes.

Two other studies (de Wit et $\mathrm{al}^{37}$ and Chung et $\mathrm{al}^{31}$ ) also hint at the interactions between neurodevelopmental and disease processes. While the results of these studies are somewhat difficult to compare with our results given differences in methodology, together they underscore the importance of accounting for age when examining neuroanatomical changes in CHR samples. Our correlational analyses of relationships between age and CT explicitly show the impact of conversion on neurodevelopmental trajectories (but see below) and, together with the examination of GAF scores and CT reductions (see Supplementary Materials) further clarify both the de Wit and the Chung results: conversion to psychosis impacts normal developmental trajectories. The role of age is succinctly and eloquently discussed in Andreou and Borgwardt's recent review. 102

However, given the relatively low $\mathrm{N}$ of $\mathrm{CHR}-\mathrm{C}$, which may have contributed to the absence of significant negative correlations in the CHR-C group in the current results, this observation will need to be replicated in a larger cohort of CHR-C.

Several limitations to this study deserve mention. First, no effects of gender were observed, although genderrelated brain differences in CHR have been reported in a study examining CT in $26 \mathrm{CHR}$ and $29 \mathrm{HC}$ using a $1.5 \mathrm{~T}$ magnet. ${ }^{103} \mathrm{It}$ is possible that the sample low $\mathrm{N}$ and low image resolution contributed to the discrepant results. Second, we did not obtain significant correlations between abnormal CT and clinical symptoms, in contrast to the Kwak et al ${ }^{44}$ which assessed 74 CHR ( 6 converters) and $34 \mathrm{HC}$. The source of these differences remains unclear, though the current study, with 152 CHR (22 converters) and $92 \mathrm{HC}$, is the largest to date conducted in an Asian population both in terms of overall sample size and number of converters. Differences in sample characteristics ${ }^{29,30,43,44}$ might have contributed to the different outcomes. Third, the age analysis in relation to CT was cross-sectional rather than longitudinal. We will follow-up this cohort longitudinally at 2 and 3 years postbaseline to assess CT changes over time in individual subjects. Fourth, our CHR sample was assessed clinically at a 1-year follow-up, which means that some CHR-NC subjects will likely convert to psychosis after the 1-year and alter observed relationships between the CHR-NC, CHR-C, and HC groups. Fifth, while functional connectivity studies have reported specific abnormalities in psychotic populations ${ }^{13}$ that do not necessarily overlap with abnormalities reported here in converters, we note that those findings apply to older populations while the CHR group studied here was young and still maturing. In summary, these novel data indicate that $\mathrm{CT}$ reductions contribute to volumetric reductions assessed in a large MRI study sample of CHR drawn from an Asian population. Our data indicate for the first time that CT reductions span a set of brain regions that largely overlap with the fronto-parietal network whose abnormalities characterize older populations with established, mixed psychiatric diagnosis. ${ }^{12,13,68}$ These data support our hypothesis of a set of cortical abnormalities common to all CHRs, and of a second set of abnormalities that are characteristic of converters to psychosis relative to non-converters and to $\mathrm{HC}$.

\section{Supplementary Material}

Supplementary material is available at Schizophrenia Bulletin online.

\section{Acknowledgments}

This work was conducted with support from Harvard Catalyst | The Harvard Clinical and Translational Science Center (National Center for Advancing Translational Sciences, National Institutes of Health Award UL 1TR002541) and financial contributions from Harvard University and its affiliated academic healthcare centers. The content is solely the responsibility of the authors and does not necessarily represent the official views of Harvard Catalyst, Harvard University and its affiliated academic healthcare centers, or the National Institutes of Health. The Authors have declared that there are no conflicts of interest in relation to the subject of this study.

\section{Funding}

This work was supported by the National Institute of Mental Health (R01 MH111448) and by R21MH109819 to E.C.D.R.

\section{References}

1. Shenton ME, Dickey CC, Frumin M, McCarley RW. A review of MRI findings in schizophrenia. Schizophr Res. 2001;49(1-2):1-52.

2. Takayanagi Y, Takahashi T, Orikabe L, et al. Classification of first-episode schizophrenia patients and healthy subjects by automated MRI measures of regional brain volume and cortical thickness. PLoS One. 2011;6(6):e21047. doi:10.1371/ journal.pone.0021047

3. Ellison-Wright I, Glahn DC, Laird AR, Thelen SM, Bullmore E. The anatomy of first-episode and chronic 
schizophrenia: an anatomical likelihood estimation metaanalysis. Am J Psychiatry. 2008;165(8):1015-1023.

4. Vita A, De Peri L, Deste G, Sacchetti E. Progressive loss of cortical gray matter in schizophrenia: a meta-analysis and meta-regression of longitudinal MRI studies. Transl Psychiatry. 2012;2:e190.

5. Ohtani T, Del Re E, Levitt JJ, et al. Progressive symptomassociated prefrontal volume loss occurs in first-episode schizophrenia but not in affective psychosis. Brain Struct Funct. 2018;223(6):2879-2892. doi:10.1007/s00429-018-1634-0

6. Lee TY, Kwon JS. Psychosis research in Asia: advantage from low prevalence of cannabis use. NPJ Schizophr. 2016;2:1.

7. Cavelti M, Kircher T, Nagels A, Strik W, Homan P. Is formal thought disorder in schizophrenia related to structural and functional aberrations in the language network? A systematic review of neuroimaging findings. Schizophr Res. 2018;199:2-16.

8. Sumner PJ, Bell IH, Rossell SL. A systematic review of taskbased functional neuroimaging studies investigating language, semantic and executive processes in thought disorder. Neurosci Biobehav Rev. 2018;94:59-75.

9. Dietsche B, Kircher T, Falkenberg I. Structural brain changes in schizophrenia at different stages of the illness: a selective review of longitudinal magnetic resonance imaging studies. Aust N Z J Psychiatry. 2017;51(5):500-508.

10. Torres US, Duran FL, Schaufelberger MS, et al. Patterns of regional gray matter loss at different stages of schizophrenia: a multisite, cross-sectional VBM study in first-episode and chronic illness. Neuroimage Clin. 2016;12:1-15.

11. Cobia DJ, Smith MJ, Wang L, Csernansky JG. Longitudinal progression of frontal and temporal lobe changes in schizophrenia. Schizophr Res. 2012;139(1-3):1-6.

12. Baker JT, Holmes AJ, Masters GA, et al. Disruption of cortical association networks in schizophrenia and psychotic bipolar disorder. JAMA Psychiatry. 2014;71(2):109-118.

13. Baker JT, Dillon DG, Patrick LM, et al. Functional connectomics of affective and psychotic pathology. Proc Natl Acad Sci U S A. 2019;116(18):9050-9059.

14. Fusar-Poli P. Predicting the development of schizophrenia. Br J Psychiatry. 2012;200(3):254-255; author reply 255.

15. Fusar-Poli P, Deste G, Smieskova R, et al. Cognitive functioning in prodromal psychosis: a meta-analysis. Arch Gen Psychiatry. 2012;69(6):562-571.

16. Fusar-Poli P, Bechdolf A, Taylor MJ, et al. At risk for schizophrenic or affective psychoses? A meta-analysis of DSM/ ICD diagnostic outcomes in individuals at high clinical risk. Schizophr Bull. 2013;39(4):923-932. doi:10.1093/schbul/sbs060

17. Li H, Zhang $\mathrm{T}, \mathrm{Xu} \mathrm{L}$, et al. A comparison of conversion rates, clinical profiles and predictors of outcomes in two independent samples of individuals at clinical high risk for psychosis in China. Schizophr Res. 2017;197:509-515. doi:10.1016/j.schres.2017.11.029

18. Blokland GAM, Mesholam-Gately RI, Toulopoulou T, et al. Heritability of neuropsychological measures in schizophrenia and nonpsychiatric populations: a systematic review and meta-analysis. Schizophr Bull. 2017;43(4):788-800.

19. Blokland GAM, Del Re EC, Mesholam-Gately RI, et al. The Genetics of Endophenotypes of Neurofunction to Understand Schizophrenia (GENUS) consortium: a collaborative cognitive and neuroimaging genetics project. Schizophr Res. 2018;195:306-317.

20. Li X, Wu K, Zhang Y, Kong L, Bertisch H, DeLisi LE. Altered topological characteristics of morphological brain network relate to language impairment in high genetic risk subjects and schizophrenia patients. Schizophr Res. 2019;208:338-343.

21. Birnbaum R, Weinberger DR. Genetic insights into the neurodevelopmental origins of schizophrenia. Nat Rev Neurosci. 2017;18(12):727-740.

22. Li M, Wang $\mathrm{Y}$, Zheng XB, et al. Meta-analysis and brain imaging data support the involvement of VRK2 (rs2312147) in schizophrenia susceptibility. Schizophr Res. 2012;142(1-3):200-205.

23. Egan MF, Goldberg TE, Gscheidle T, et al. Relative risk for cognitive impairments in siblings of patients with schizophrenia. Biol Psychiatry. 2001;50(2):98-107.

24. Brent BK, Thermenos HW, Keshavan MS, Seidman LJ. Gray matter alterations in schizophrenia high-risk youth and earlyonset schizophrenia: a review of structural MRI findings. Child Adolesc Psychiatr Clin N Am. 2013;22(4):689-714.

25. Fusar-Poli P, Borgwardt S, Crescini A, et al. Neuroanatomy of vulnerability to psychosis: a voxel-based meta-analysis. Neurosci Biobehav Rev. 2011;35(5):1175-1185.

26. Sun D, Phillips L, Velakoulis D, et al. Progressive brain structural changes mapped as psychosis develops in "at risk" individuals. Schizophr Res. 2009;108(1-3):85-92. doi:10.1016/j. schres.2008.11.026

27. Pantelis C, Yücel M, Wood SJ, McGorry PD, Velakoulis D. Early and late neurodevelopmental disturbances in schizophrenia and their functional consequences. Aust $N Z J$ Psychiatry. 2003;37(4):399-406.

28. Borgwardt SJ, McGuire PK, Aston J, et al. Reductions in frontal, temporal and parietal volume associated with the onset of psychosis. Schizophr Res. 2008;106(2-3):108-114. doi:10.1016/j.schres.2008.08.007

29. Klauser P, Zhou J, Lim JKW, et al. Lack of evidence for regional brain volume or cortical thickness abnormalities in youths at clinical high risk for psychosis: findings from the longitudinal youth at risk study. Schizophr Bull. 2015;41(6):1285-1293. doi:10.1093/schbul/sbv012

30. Cannon TD, Chung Y, He G, et al. Progressive reduction in cortical thickness as psychosis develops: a multisite longitudinal neuroimaging study of youth at elevated clinical risk. Biol Psychiatry. 2015;77(2):147-157. doi:10.1016/j. biopsych.2014.05.023

31. Chung Y, Allswede D, Addington J, et al. Cortical abnormalities in youth at clinical high-risk for psychosis: findings from the NAPLS2 cohort. Neuroimage Clin. 2019;23:101862.

32. Buchy L, Stowkowy J, MacMaster FP, Nyman K, Addington J. Meta-cognition is associated with cortical thickness in youth at clinical high risk of psychosis. Psychiatry Res. 2015;233(3):418-423.

33. Fornito A, Yung AR, Wood SJ, et al. Anatomic abnormalities of the anterior cingulate cortex before psychosis onset: an MRI study of ultra-high-risk individuals. Biol Psychiatry. 2008;64(9):758-765.

34. Sprooten E, Papmeyer M, Smyth AM, et al. Cortical thickness in first-episode schizophrenia patients and individuals at high familial risk: a cross-sectional comparison. Schizophr Res. 2013;151(1-3):259-264.

35. Bartholomeusz CF, Cropley VL, Wannan C, Di Biase M, McGorry PD, Pantelis C. Structural neuroimaging across early-stage psychosis: Aberrations in neurobiological trajectories and implications for the staging model. Aust $N Z J$ Psychiatry. 2017;51(5):455-476.

36. Takayanagi Y, Kulason S, Sasabayashi D, et al. Reduced thickness of the anterior cingulate cortex in individuals with 
an at-risk mental state who later develop psychosis. Schizophr Bull. 2017;43(4):907-913.

37. de Wit S, Wierenga LM, Oranje B, et al. Brain development in adolescents at ultra-high risk for psychosis: Longitudinal changes related to resilience. Neuroimage Clin. 2016;12:542-549.

38. Panizzon MS, Fennema-Notestine C, Eyler LT, et al. Distinct genetic influences on cortical surface area and cortical thickness. Cereb Cortex. 2009;19(11):2728-2735.

39. Vijayakumar N, Allen NB, Youssef G, et al. Brain development during adolescence: A mixed-longitudinal investigation of cortical thickness, surface area, and volume. Human Brain Mapping. 2016;37(6):2027-2038. doi:10.1002/hbm.23154

40. Tamnes CK, Herting MM, Goddings A-L, et al. Development of the cerebral cortex across adolescence: a multisample study of inter-related longitudinal changes in cortical volume, surface area, and thickness. $J$ Neurosci. 2017;37(12):3402-3412. doi:10.1523/JNEUROSCI.3302-16.2017

41. Benetti S, Pettersson-Yeo W, Hutton C, et al. Elucidating neuroanatomical alterations in the at risk mental state and first episode psychosis: a combined voxel-based morphometry and voxel-based cortical thickness study. Schizophr Res. 2013;150(2-3):505-511.

42. Tognin S, Riecher-Rössler A, Meisenzahl EM, et al. Reduced parahippocampal cortical thickness in subjects at ultra-high risk for psychosis. Psychol Med. 2014;44(3):489-498.

43. Jung WH, Kim JS, Jang JH, et al. Cortical thickness reduction in individuals at ultra-high-risk for psychosis. Schizophr Bull. 2011;37(4):839-849.

44. Kwak YB, Kim M, Cho KIK, Lee J, Lee TY, Kwon JS. Reduced cortical thickness in subjects at clinical high risk for psychosis and clinical attributes. Aust N Z P Psychiatry. 2019;53(3):219-227.

45. Jung WH, Jang JH, Shin NY, et al. Regional brain atrophy and functional disconnection in Broca's area in individuals at ultra-high risk for psychosis and schizophrenia. PLoS One. 2012;7(12):e51975.

46. Gifford G, Crossley N, Fusar-Poli P, et al. Using neuroimaging to help predict the onset of psychosis. Neuroimage. 2017;145(Pt B):209-217.

47. Mirzaalian H, Ning L, Savadjiev P, et al. Inter-site and interscanner diffusion MRI data harmonization. Neuroimage. 2016;135:311-323.

48. Cetin Karayumak S, Bouix S, Ning L, et al. Retrospective harmonization of multi-site diffusion MRI data acquired with different acquisition parameters. Neuroimage. 2019;184:180-200.

49. Rosenstein M, Foltz PW, DeLisi LE, Elvevåg B. Language as a biomarker in those at high-risk for psychosis. Schizophr Res. 2015;165(2-3):249-250.

50. Corcoran CM, Carrillo F, Fernández-Slezak D, et al. Prediction of psychosis across protocols and risk cohorts using automated language analysis. World Psychiatry. 2018;17(1):67-75.

51. Kuperberg GR. Language in schizophrenia Part 1: an introduction. Lang Linguist Compass. 2010;4(8):576-589.

52. Mesholam-Gately RI, Giuliano AJ, Goff KP, Faraone SV, Seidman LJ. Neurocognition in first-episode schizophrenia: a meta-analytic review. Neuropsychology. 2009;23(3):315-336.

53. Rabagliati H, Delaney-Busch N, Snedeker J, Kuperberg G. Spared bottom-up but impaired top-down interactive effects during naturalistic language processing in schizophrenia: evidence from the visual-world paradigm. Psychol Med. 2019;49(8):1335-1345.

54. Fedorenko E, Thompson-Schill SL. Reworking the language network. Trends Cogn Sci. 2014;18(3):120-126.

55. Friederici AD. Evolution of the neural language network. Psychon Bull Rev. 2017;24(1):41-47.

56. Hagoort $\mathrm{P}$. The core and beyond in the language-ready brain. Neurosci Biobehav Rev. 2017;81(Pt B):194-204.

57. Hagoort P. The neurobiology of language beyond singleword processing. Science. 2019;366(6461):55-58.

58. Nasios G, Dardiotis E, Messinis L. From Broca and Wernicke to the neuromodulation era: insights of brain language networks for neurorehabilitation. Behav Neurol. 2019;2019:9894571.

59. Rezaii N, Walker E, Wolff P. A machine learning approach to predicting psychosis using semantic density and latent content analysis. NPJ Schizophr. 2019;5(1):9.

60. Ratana R, Sharifzadeh H, Krishnan J, Pang S. A Comprehensive review of computational methods for automatic prediction of schizophrenia with insight into indigenous populations. Front Psychiatry. 2019;10:659.

61. Palaniyappan L, Mota NB, Oowise S, et al. Speech structure links the neural and socio-behavioural correlates of psychotic disorders. Prog Neuropsychopharmacol Biol Psychiatry. 2019;88:112-120.

62. Zhang T, Li H, Woodberry KA, et al. Prodromal psychosis detection in a counseling center population in China: an epidemiological and clinical study. Schizophr Res. 2014;152(23):391-399. doi:10.1016/j.schres.2013.11.039

63. Zheng L, Wang J, Zhang T, Li H, Li C, Jiang K. The Chinese version of the SIPS/SOPS: a pilot study of reliability and validity. Chinese Mental Health Journal. 2012;26(8):571-576.

64. Del Re EC, Gao Y, Eckbo R, et al. A new MRI masking technique based on multi-atlas brain segmentation in controls and schizophrenia: a rapid and viable alternative to manual masking. J Neuroimaging. 2016;26(1):28-36.

65. Fischl B, Salat DH, Busa E, et al. Whole brain segmentation: automated labeling of neuroanatomical structures in the human brain. Neuron. 2002;33(3):341-355.

66. Miller TJ, McGlashan TH, Rosen JL, et al. Prodromal assessment with the structured interview for prodromal syndromes and the scale of prodromal symptoms: predictive validity, interrater reliability, and training to reliability. Schizophr Bull. 2003;29(4):703-715.

67. Tu PC, Lee YC, Chen YS, Li CT, Su TP. Schizophrenia and the brain's control network: aberrant within- and betweennetwork connectivity of the frontoparietal network in schizophrenia. Schizophr Res. 2013;147(2-3):339-347.

68. Marek S, Dosenbach NUF. The frontoparietal network: function, electrophysiology, and importance of individual precision mapping. Dialogues Clin Neurosci. 2018;20(2):133-140.

69. Witthaus H, Kaufmann C, Bohner G, et al. Gray matter abnormalities in subjects at ultra-high risk for schizophrenia and first-episode schizophrenic patients compared to healthy controls. Psychiatry Res. 2009;173(3):163-169.

70. Mechelli A, Riecher-Rössler A, Meisenzahl EM, et al. Neuroanatomical abnormalities that predate the onset of psychosis: a multicenter study. Arch Gen Psychiatry. 2011;68(5):489-495.

71. Cropley VL, Lin A, Nelson B, et al. Baseline grey matter volume of non-transitioned "ultra high risk" for psychosis individuals with and without attenuated psychotic symptoms 
at long-term follow-up. Schizophr Res. 2016;173(3):152-158. doi:10.1016/j.schres.2015.05.014

72. Friederici AD, Rüschemeyer SA, Hahne A, Fiebach CJ. The role of left inferior frontal and superior temporal cortex in sentence comprehension: localizing syntactic and semantic processes. Cereb Cortex. 2003;13(2):170-177.

73. Friederici AD, Gierhan SM. The language network. Curr Opin Neurobiol. 2013;23(2):250-254.

74. Chai LR, Mattar MG, Blank IA, Fedorenko E, Bassett DS. Functional network dynamics of the language system. Cereb Cortex. 2016;26(11):4148-4159.

75. Alho K, Rinne T, Herron TJ, Woods DL. Stimulus-dependent activations and attention-related modulations in the auditory cortex: a meta-analysis of fMRI studies. Hear Res. 2014;307:29-41.

76. Walenski M, Europa E, Caplan D, Thompson CK. Neural networks for sentence comprehension and production: an ALE-based meta-analysis of neuroimaging studies. Hum Brain Mapp. 2019;40(8):2275-2304.

77. Kroczek LOH, Gunter TC, Rysop AU, Friederici AD, Hartwigsen G. Contributions of left frontal and temporal cortex to sentence comprehension: evidence from simultaneous TMS-EEG. Cortex. 2019;115:86-98.

78. Natsubori T, Hashimoto R, Yahata N, et al. An fMRI study of visual lexical decision in patients with schizophrenia and clinical high-risk individuals. Schizophr Res. 2014;157(1-3):218-224.

79. Sabb FW, van Erp TG, Hardt ME, et al. Language network dysfunction as a predictor of outcome in youth at clinical high risk for psychosis. Schizophr Res. 2010;116(2-3):173-183.

80. Thermenos HW, Whitfield-Gabrieli S, Seidman LJ, et al. Altered language network activity in young people at familial high-risk for schizophrenia. Schizophr Res. 2013;151(1-3):229-237.

81. Smith K, Azami H, Escudero J, Parra MA, Starr JM. Comparison of network analysis approaches on EEG connectivity in beta during visual short-term memory binding tasks. Conf Proc IEEE Eng Med Biol Soc. 2015;2015:22072210. doi:10.1109/EMBC.2015.7318829

82. Finn ES, Shen X, Scheinost D, et al. Functional connectome fingerprinting: identifying individuals using patterns of brain connectivity. Nat Neurosci. 2015;18(11):1664-1671.

83. Kong J, Jensen K, Loiotile R, et al. Functional connectivity of the frontoparietal network predicts cognitive modulation of pain. Pain. 2013;154(3):459-467.

84. Wu CY, Ho MH, Chen SH. A meta-analysis of fMRI studies on Chinese orthographic, phonological, and semantic processing. Neuroimage. 2012;63(1):381-391.

85. Seghier ML. The angular gyrus: multiple functions and multiple subdivisions. Neuroscientist. 2013;19(1):43-61.

86. Gow DW Jr. The cortical organization of lexical knowledge: a dual lexicon model of spoken language processing. Brain Lang. 2012;121(3):273-288.

87. Koreki A, Maeda T, Okimura T, et al. Dysconnectivity of the agency network in schizophrenia: a functional magnetic resonance imaging study. Front Psychiatry. 2019;10:171.
88. Andre J, Picchioni M, Zhang R, Toulopoulou T. Working memory circuit as a function of increasing age in healthy adolescence: a systematic review and meta-analyses. Neuroimage Clin. 2016;12:940-948.

89. Igelström KM, Graziano MSA. The inferior parietal lobule and temporoparietal junction: a network perspective. Neuropsychologia. 2017;105:70-83.

90. Owens MM, Gray JC, Amlung MT, Oshri A, Sweet LH, MacKillop J. Neuroanatomical foundations of delayed reward discounting decision making. Neuroimage. 2017;161:261-270.

91. Feng W, Wu Y, Jan C, Yu H, Jiang X, Zhou X. Effects of contextual relevance on pragmatic inference during conversation: an fMRI study. Brain Lang. 2017;171:52-61.

92. Grill-Spector K, Weiner KS, Kay K, Gomez J. The functional neuroanatomy of human face perception. Annu Rev Vis Sci. 2017;3:167-196.

93. Dricu M, Frühholz S. Perceiving emotional expressions in others: activation likelihood estimation meta-analyses of explicit evaluation, passive perception and incidental perception of emotions. Neurosci Biobehav Rev. 2016;71:810-828.

94. Kolodny O, Edelman S. The evolution of the capacity for language: the ecological context and adaptive value of a process of cognitive hijacking. Philos Trans R Soc Lond, B, Biol Sci. 2018;373(1743). doi:10.1098/rstb.2017.0052

95. Gogtay N, Giedd JN, Lusk L, et al. Dynamic mapping of human cortical development during childhood through early adulthood. Proc Natl Acad Sci US A. 2004;101(21):8174-8179.

96. Sowell ER, Thompson PM, Toga AW. Mapping changes in the human cortex throughout the span of life. Neuroscientist. 2004;10(4):372-392.

97. Keshavan MS, Anderson S, Pettegrew JW. Is schizophrenia due to excessive synaptic pruning in the prefrontal cortex? The Feinberg hypothesis revisited. $J$ Psychiatr Res. 1994;28(3):239-265.

98. Natu VS, Gomez J, Barnett M, et al. Apparent thinning of human visual cortex during childhood is associated with myelination. Proc Natl Acad Sci U S A. 2019;116(41):20750-20759.

99. Fjell AM, Westlye LT, Amlien I, et al. High-expanding cortical regions in human development and evolution are related to higher intellectual abilities. Cereb Cortex. 2015;25(1):2634. doi:10.1093/cercor/bht201

100. Ducharme S, Albaugh MD, Nguyen TV, et al. Trajectories of cortical thickness maturation in normal brain developmentthe importance of quality control procedures. Neuroimage. 2016;125:267-279.

101. Hogstrom LJ, Westlye LT, Walhovd KB, Fjell AM. The structure of the cerebral cortex across adult life: age-related patterns of surface area, thickness, and gyrification. Cereb Cortex. 2013;23(11):2521-2530.

102. Andreou C, Borgwardt S. Structural and functional imaging markers for susceptibility to psychosis. Mol Psychiatry. 2020:1-13. doi:10.1038/s41380-020-0679-7

103. Guma E, Devenyi GA, Malla A, Shah J, Chakravarty MM, Pruessner M. Neuroanatomical and symptomatic sex differences in individuals at clinical high risk for psychosis. Front Psychiatry. 2017;8:291. 